\title{
Indicators for Urban Green (IUG): Proposal for a Computational Implementation
}

XXIV International Conference

of the Iberoamerican Society

of Digital Graphics

Medellin | Colombia

\author{
Thayssa Barbosa da Silva Neves \\ Universidade Federal da Paraíba (UFPB) | Brasil | thay.arquitetura@hotmail.com \\ Lucila Chebel Labaki \\ Universidade Estadual de Campinas (UNICAMP) | Brasil | llabaki@gmail.com
}

\begin{abstract}
Due to the reality of the constructive densification process at urban areas in disproportion to urban vegetation cover, the aim of this paper is to formulate two indicators for measuring vegetated density. A proportion between vegetated density and built density was proposed. In order to develop a system of computational tools, an algorithmic-parametric framework called Indicators for Urban Green (IUG) was implemented. The focus is on the internal validation of the IUG through testing hypothetical urban situations. The proposed method leans on the premise of contributing to quanti-qualitative studies focused on mitigating the impacts of anthropogenic action caused at the microclimatic scale of cities.
\end{abstract}

Keywords: Urban green; urban indicators; vegetated density; built density; computational implementation.

\section{INTRODUÇÃO E MOTIVAÇÃO}

A cidade e o clima se encontram em uma dinâmica simbiótica entremeada ao espaço urbano (NEVES, 2020). Faz-se importante o estímulo da atribuição de um peso maior à questão do clima, enquanto parâmetro a ser considerado no modo de produção e planejamento urbano. O conforto ambiental gerado por ambientes construídos adaptados à realidade climática local é capaz de mitigar os impactos provocados pelo processo de urbanização (BARBIRATO et. al., 2012).

Os parâmetros de densidade construída e superfícies impermeabilizadas, são agentes contribuinte para formação de fenômenos climáticos ("ilhas de calor urbana"). Assim como, para o esgotamento do sistema de drenagem urbana, observando a maior recorrência de enchentes provocadas pelas alterações climáticas no regime de chuvas (ACCIOLY \& DAVIDSON, 1998).

Em função de métodos de mensuração do parâmetro densidade urbana, Berghauser-Pont \& Haupt (2009) propõem uma metodologia a qual estabelece um conceito produtivo de modo a permitir obter uma compreensão da forma e densidade urbana da micro à macroescala (da quadra ao bairro/cidade). Em suma, o Spacematrix é um conjunto de ferramentas que visa auxiliar a tomada de decisão e pesquisas acadêmicas voltadas para a temática de densidade urbana, oferecendo indicadores que, ao serem calculados, possibilitam compreender a tipologia da forma urbana predominante.

Com a priorização dos adensamentos vegetados na escala urbana, influencia-se nos níveis de conforto dos habitantes, produzindo melhorias para a qualidade ambiental urbana (LABAKI et. al., 2011). A partir da análise do parâmetro da vegetação, é possível correlaciona-la à densidade construída, de modo a gerar conhecimento acerca dos níveis de proporção do que se tem edificado com o que se tem (ou deveria estar) arborizado. Desta forma, a vegetação urbana, considerada como valioso recurso natural de mitigação dos efeitos das alterações climáticas (SPANGENBERG, 2009), será analisada nesta investigação em uma relação proporcional ao parâmetro de densidade edificada, para que seja possível encontrar a proporção existente entre os mesmos.

O sombreamento e a evapotranspiração produzidos pela vegetação, podem fornecer resfriamento passivo para edificações próximas, pois as folhas são capazes de absorver $90 \%$ da radiação visível e $60 \%$ da infravermelha (LABAKI et. al., 2011). Quanto a proporção mínima de cobertura vegetal das cidades, Oke (1973 apud LOMBARDO, 1985; NUCCI, 2001; FERREIRA et. al., 2019 ) indica que esta precisa ocupar $30 \%$ do seu território, para que se mantenha adequado o balanço térmico, somente a Região Administrativa Central que estaria isenta desta indicação, por ser uma área consolidada, o estudo de inserção de verde deveria ser diferenciado das outras Regiões Administrativas.

Para quantificar a cobertura vegetal, alguns autores recorrem à formulação de índices de áreas verdes, que buscam estabelecer uma relação entre a quantificação total das áreas vegetadas (espaços livres públicos/privados) em proporção a outros atributos como área total de estudo, elementos arbóreos ou habitantes $/ \mathrm{m}^{2}$ (ARAÚJO e FERREIRA, 2014; CALLEJAS et al., 2012; HARDER et al., 2006; ROSSET, 2005).

O termo "densidade" se exprime como a relação existente entre os elementos arbóreos, e a "disposição dos indivíduos" identifica como as árvores estão adensadas, numa visão mais genérica do conjunto (PEIXOTO et. al., 1999 apud LABAKI et. al., 2011). Logo, torna-se essencial observar, dentro dos tipos de áreas verdes existentes, a 
tipologia de árvores predominante, pois os portes dos elementos arbóreos devem corroborar para a função da área verde na qual os mesmos estão inseridos, assim como levar em consideração a seu padrão de adensamento, para que se alcance maiores índices de qualidade ambiental (LABAKI et. al., 2011).

Considerando a cidade como um sistema complexo (BEIRÃO, 2012), esta pesquisa explora formas nãoconvencionais de implementação do espaço urbano que sejam veiculadas por ferramentas, como as que apresentam sua essência sob a lógica algorítmica e a modelagem paramétrica, pois apresentam maior agilidade no que tange à manipulação dos dados de entrada solicitados para análise e desempenho.

Por fim, frente o exposto, urge a observância de atributos urbanísticos voltados para uso do solo à luz de um embasamento e preocupação acerca da qualidade ambiental, de vida e climática das cidades e dos habitantes. Norteando a pesquisa, no que tange ao enfoque escolhido, esta se divide em 2 temáticas interseccionada por uma $3^{a}$ subsidiária para 0 desenvolvimento da metodologia proposta (fig. 1).

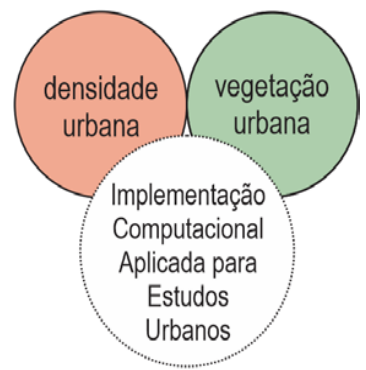

Figura 1: Articulação das temáticas abordadas.

\section{OBJETIVOS E METODOLOGIA}

Frente as problemáticas levantadas, e a definição da linha temática como embasamento teórico, os objetivos desta pesquisa se estabelecem, de modo geral, em estabelecer uma relação de proporção de parâmetros urbanísticos comprovadamente influenciadores na escala climática da urbe: sendo estes os de densidade urbana e vegetação urbana, por meio de uma abordagem computacional que siga a lógica algorítmica e a modelagem paramétrica. Para tal, buscou-se elaborar dois indicadores que permitam mensurar e ponderar a quantidade e a proporção de "densidade vegetada" em dado recorte urbano, assim como relacionar este atributo proporcionalmente à "densidade construída" calculada existente. De modo que sejam implementados computacionalmente, desenvolvendo-se um sistema de suporte ao planejamento urbano, utilizando-se de recursos algorítmicos-paramétricos.

Esta é uma pesquisa por simulação de natureza aplicada, se utiliza de conhecimentos gerados pela pesquisa básica somada à tecnologia existente, de acordo com a definição de Prodanov \& Freitas (2013). Portanto, os procedimentos metodológicos (fig.2) se destrincham nas seguintes fases:

I. Recorte do problema na base teórica levantada;

II. Proposição de indicadores de modo que possam quanti-qualificar os atributos urbanísticos em questão desta pesquisa (densidades construída e vegetada) e formulação de uma metodologia para aplicá-los e analisá-los;

III. Elaboração de uma implementação computacional para os indicadores por meio do uso do plugin Grasshopper inserido no software Rhinoceros;

IV. Aplicação da implementação computacional para compreensão do comportamento dos indicadores em Testes Hipotéticos (visualizando os cenários reais), subdivididos em subáreas para visualização dos cenários reais;

V. Processo de análise dos resultados e proposta de encaminhamentos;

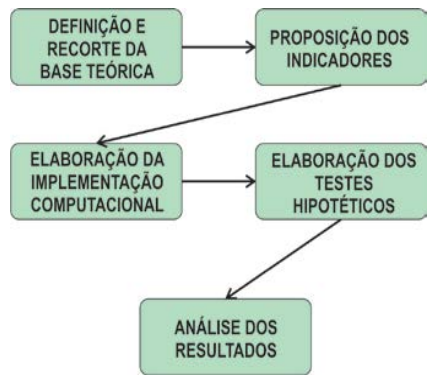

Figura 2: Procedimentos metodológicos em fluxograma.

Foram elaborados quatro Testes Hipotéticos, estabelecidos de modo a seguir uma lógica de equidade ou aproximação do tamanho área total do recorte urbano, desta forma pôde-se aumentar o poder comparativo entre os mesmos. Os Testes Hipotéticos foram subdivididos em sub-testes de maneira a alterar os dados de densidade construída e os de densidade vegetada - tipologias estas que serão explanadas na seção a seguir - e viabilizar uma maior gama de resultados e compreensão dos valores encontrados (fig.3).

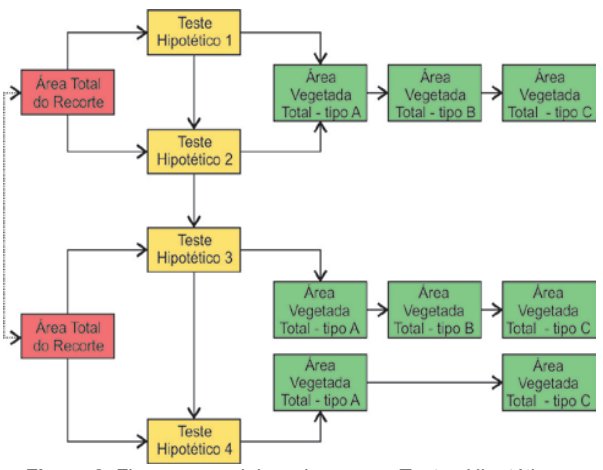

Figura 3: Fluxograma elaborado para os Testes Hipotéticos. 


\section{ELABORAÇÃO DOS INDICADORES}

Segundo alguns autores (DUARTE, 2000; SAMPAIO, 1981; GOMES \& LAMBERTS, 2009), a correlação de parâmetros urbanísticos concernentes à ocupação do solo e áreas permeáveis podem fornecer dados de fundamental importância para alcançar maiores níveis de amenização do rigor climático que incide gradualmente nos grandes núcleos urbanos, assolados cada vez mais por problemáticas urbanas como enchentes e formação de "ilhas de calor" que possui como um dos gatilhos os altos índices de superfície permeabilizada em desproporção à presença de áreas verdes/arborização de vias (Autor, 2020).

Para proposição dos indicadores, levou-se em consideração outras pesquisas que seguiram um viés semelhante, como o modelo empírico de cálculo de proporção entre padrões de ocupação do solo e superfícies verde e de água formulado por Duarte (2000), os índices de áreas verdes formulados para quantificação de cobertura vegetal (ARAÚJO \& FERREIRA, 2014; HARDER, RIBEIRO \& TAVARES, 2005; ROSSET, 2005; CALLEJAS et. al., 2012; HENKE-OLIVEIRA, 1996), tanto quanto o uso da lógica algorítmico-paramétrica para estudos de planejamento urbano por meio de simulação computacional (LIMA, 2017).

\section{IDVAT - INDICADOR DE DENSIDADE VEGETADA POR ÁREA TOTAL}

O termo "densidade vegetada" é definido pelas formas de adensamento arbóreo predominante de acordo com a definição de Labaki et. al. (2011). Portanto, nesta pesquisa, as áreas verdes são consideradas tendo em vista os agrupamentos arbóreos com maior predominância (quadro 1).

\begin{tabular}{|c|c|c|c|}
\hline $\begin{array}{l}\text { TIPOS DE } \\
\text { CONCENTRAÇÃO } \\
\text { ARBÓREA }\end{array}$ & PORTE & $\begin{array}{c}\text { TIPOS } \\
\text { ARBÓREOS E } \\
\text { ALTURA } \\
\text { ESTIMADA }\end{array}$ & $\begin{array}{c}\text { TIPOLOGIA DA } \\
\text { COPA }\end{array}$ \\
\hline & PEQUENO & $\begin{array}{c}\text { Rasteira; } \\
\text { Arbustiva baixa }\end{array}$ & $\begin{array}{c}\text { Não gera } \\
\text { sombreamento }\end{array}$ \\
\hline & MÉDIO & $\begin{array}{l}\text { Arbustiva alta; } \\
\text { Árvores com até } \\
\text { aprox. } 5 \text { à } 6 \\
\text { metros de altura }\end{array}$ & $\begin{array}{c}\text { Não gera } \\
\text { sombreamento } \\
\text { suficiente para o } \\
\text { pedestre }\end{array}$ \\
\hline & GRANDE & $\begin{array}{l}\text { Árvores maiores } \\
\text { que } 7 \text { metros de } \\
\text { altura (aprox.) }\end{array}$ & $\begin{array}{c}\text { Gera } \\
\text { sombreamento } \\
\text { suficiente para o } \\
\text { pedestre }\end{array}$ \\
\hline
\end{tabular}

Quadro 1: Definição dos tipos de concentração arbórea e respectivas classificações quanto ao porte, altura estimada e tipologia da copa.

Basicamente, este indicador tem como objetivo apresentar a proporção existente de densidade vegetada dentro de uma região urbana delimitada. A proposta é, ademais quantificar a cobertura vegetal presente, propor ponderá-la de acordo com o tipo arbóreo predominante em cada uma (eq.1).

\section{IDVAT $=$}

$\underline{\left(\sum A V \text { 's tipo } A \times p_{x}\right)+\left(\sum A V \text { Is tipo } B \times p_{y}\right)+\left(\sum A V \text { 's tipo } C \times p_{z}\right)}$ Área Total do Recorte Urbano
Onde:

$A V=$ Área Vegetada

$p=$ peso atribuído

$x=$ predominância arbórea tipo $A$

$y=$ predominância arbórea tipo $B$

$\mathrm{z}=$ predominância arbórea tipo $\mathrm{C}$

\section{IDVDC - INDICADOR DE DENSIDADE VEGETADA POR DENSIDADE CONSTRUÍDA}

Algumas pesquisas da área de climatologia urbana defendem, unanimemente, a tese de que considerar a proporção do verde quantificado pela densidade construída apresenta maior eficiência no que tange a correlação de regiões urbanas desprovidas, ou com mínima presença de cobertura vegetal, apresentarem um maior poder de incremento das temperaturas ( $\mathrm{NUCCl}$, 2001; FERREIRA, 2019; FERREIRA et. al., 2019; SILVA et. al., 2015; ABREU \& LABAKI, 2010; GOMES \& LAMBERTS, 2009; DUARTE, 2000).

Diante disso, complementando o IDVAT, esta investigação propõe o Indicador de Densidade Vegetada e Densidade Construída (IDVDC), objetivando estabelecer uma correlação entre a mensuração da densidade construída e da densidade vegetada em um dado espaço urbano (eq. 3). Tendo por base o modelo empírico formulado por Duarte (2000), no qual a autora estabeleceu um índice considerando dados de uso e ocupação do solo, cobertura vegetal e corpos d'água.

O modo de quantificação do adensamento construtivo se utiliza do indicador FSI (Building Intensity) (eq. 2), formulado por Berghauser-Pont \& Haupt (2009), como meio de mensurar a área total construída das edificações, no Brasil, esse indicador se assemelha ao coeficiente de aproveitamento.

$$
\begin{gathered}
\boldsymbol{F S I}=\frac{\sum \text { Área Construída Total }}{\text { Área Total do Recorte Urbano }} \\
\text { IDVDC }=\frac{I D V A T}{F S I}
\end{gathered}
$$

\section{ELABORAÇÃO DA IMPLEMENTAÇÃO COMPUTACIONAL}

A formulação do conjunto de ferramentas proposto tem como base uma linha de pensamento que norteou todo o processo de elaboração ferramental, adotando-se a lógica algorítmica-paramétrica, na qual aplica-se estratégias computacionais como a Linguagem de Programação Visual (LVP) - Visual Programming Language (VPL) - e Sistemas Generativos (SG) - Generative Systems (GS).

A LVP pode ser compreendida como uma interface mais simplificada para programação por meio do uso de comandos arranjados visualmente para transmitir uma função-objetivo (LIMA, 2017). Se caracteriza por elaborar sistemas "box-and-wire", no qual pode ser digitado o código para a ação desejada, permitindo que se organize 
o campo visual das ações (canva) hierarquicamente (CELANI e VAZ, 2012)

O SG é um meio pelo qual se busca solucionar problemáticas similares em diversos tipos de situações (CELANI, 2011). A formulação de sistemas generativos permite que se crie um projeto mais genérico no intuito de que, a partir deste, encontre-se mais possibilidade de soluções para contextos distintos. Gerando mais opções de resultados, esse modo de projeto/análise pode ser utilizado com a função de otimização (convergência) ou variedade (divergência) (CELANI, 2011). O procedimento metodológico dos sistemas generativos possui sequências de instruções chamadas de algoritmo ou estratégias algorítmicas.

Diante da abordagem breve acerca das estratégias computacionais apresentadas, esta investigação propõe a criação da implementação dos indicadores aqui propostos (IDVAT e IDVDC), corroborando para a criação do Indicadores para o Verde Urbano (IVU). Este é definido como um sistema de ferramentas computacionais que, ao indicar a criação de algoritmos que contenham a memória de cálculo dos indicadores propostos nesta pesquisa, auxiliem pesquisadores e planejadores na análise dos parâmetros urbanos de enfoque, sob uma abordagem algorítmica-paramétrica, promovendo a possibilidade de uma avaliação multicenários em tempo otimizado.

$\mathrm{Na}$ perspectiva de Lima (2017), um SG pode ser compreendido como "uma estratégia algorítmica (pois funciona sob a perspectiva do atendimento a uma sequência de instruções), paramétrica (pois pode ser articulado por meio da variação de um ou mais parâmetros) e que tem por objetivo fornecer uma série de soluções diferentes de acordo com as variações de seus parâmetros geradores" (LIMA, 2017).

O procedimento do IVU estrutura-se, basicamente, em duas etapas (fig.4). Na primeira etapa, é ajustada a base cartográfica para a locação das edificações e a contabilização do número de pavimentos das mesmas. $\mathrm{Na}$ segunda etapa, é realizado a manipulação dos dados de entrada (inputs) para geração dos dados de saída (outputs) (fig. 4). Sendo estes definidos da seguinte forma:

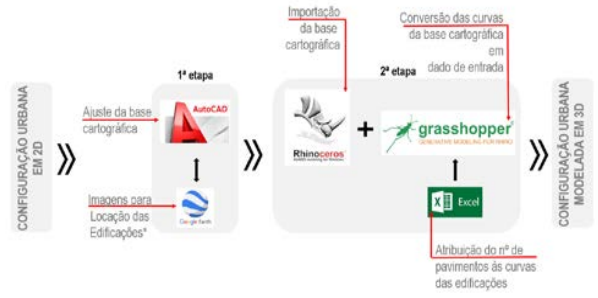

Figura 4: Fluxograma esquemático da implementação computacional: visão inter-ferramental.

Os dados de entrada a serem trabalhados no procedimento de implementação são estes:

A) Атотац: todo o perímetro de estudo é considerado como Área Total. É calculado por um comando no Grasshopper selecionando todo a camada (layer) da perimetral;
B) AVTOTAL: área vegetada total selecionada $\left(\mathrm{em} \mathrm{m}^{2}\right)$ em domínios públicos e privados (canteiros centrais, praças, ZPA's, parques, cemitérios, áreas verdes de condomínios, quintais, tetos e paredes verdes);

C) AVtotal tipo A: total de área vegetada do tipo A, selecionando todas as áreas verdes com predominância arbórea de grande porte, assim como árvores isoladas de áreas públicas e privadas;

D) AVtotal tipo B: total de área vegetada do tipo $B$, selecionando todas as áreas verdes com predominância arbórea-arbustiva de médio porte;

E) AVtotAL TIPo c: total de área vegetada do tipo $C$, selecionando todas as áreas verdes com predominância arbustiva de pequeno porte, assim como áreas gramadas.

Os dados acima, após serem combinados, são capazes de gerar: o IDVAT; os indicadores formulados por Berghauser-Pont e Haupt (2009), implementados computacionalmente por Lima (2017), dos quais se utiliza apenas o FSI; e o IDVDC (fig. 5).

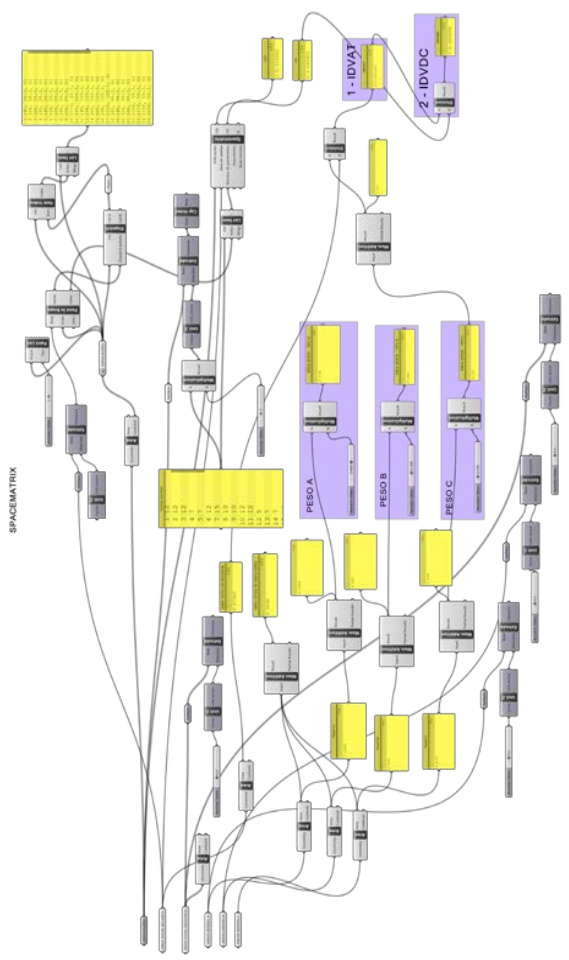

Figura 5: Esquema da implementação computacional: algoritmos elaborados no Grasshopper para implementação do IVU.

\section{ENSAIO PRELIMINAR: TESTES HIPOTÉTICOS}

Foram elaborados quatro Testes Hipotéticos, de modo a contribuir para a validação interna dos indicadores propostos. Primeiramente, formulou-se os Testes 
Hipotéticos 1 e 2, nos quais buscou-se manter iguais os tamanhos dos recortes urbanos e as áreas vegetadas, alterando apenas o padrão de adensamento construtivo. A partir do valor do tamanho da Área Total, buscou-se elaborar os testes 3 e 4 com valores aproximados em situações urbanas distintas.

Para as amostragens hipotéticas, descreve-se a seguinte sequência:

1) Diretamente no Rhinoceros, elaborar uma planta baixa com um conjunto de quatro quadras com lotes $e$ edificações iguais;

2) Organizar os layers do recorte urbano no Rhinoceros de forma que sigam, preferencialmente, as seguintes nomenclaturas: "LIMITE DO RECORTE", "ÁREA TOTAL", "ÁREA VEGETADA TIPO A", "ÁREA VEGETADA TIPO B", "ÁREA VEGETADA TIPO C" e "EDIFICAÇÕES";

3) Abrir o Grasshopper e o arquivo-base do IVU;

4) Identificar as curvas das EDIFICAÇÕES, ÁREA TOTAL DO RECORTE, ÁREA TOTAL VEGETADA, ÁREA VEGETADA TIPO A, ÁREA VEGETADA TIPO B e ÁREA VEGETADA TIPO C, desenhadas no Rhinoceros, atribuindo-os como "curvas", uma por uma, no Grasshopper,

5) Com o auxílio do Excel, associar o número de pavimentos contabilizados na etapa 4 às curvas das edificações enumeradas pelo Grasshopper (podendo ser uma numeração randômica ou ordenada, depende da forma como se seleciona as curvas);

6) Copiar o número de pavimentos contabilizados no Excel para o painel destinado para tal no algoritmo do Spacematrix no sistema generativo do IVU no Grasshopper.

7) Gerar os dados finais.

\section{TESTE HIPOTÉTICO 1}

O primeiro teste foi esboçado sobre uma área total de 2,7 ha, sendo destes 0,3 ha de áreas vegetadas. O adensamento construtivo predominante é de "baixa densidade", definindo-se de 1 a 5 o número de pavimentos (fig. 6). Para validação do teste, elaborou-se 4 sub-testes, nos quais $01^{\circ}$ sub-teste considerou apenas as áreas vegetadas com predominância arbórea do tipo $A$, no $2^{\circ}$ sub-teste todas tipo $\mathrm{B}$, no $3^{\circ}$ todas tipo $\mathrm{C}$, no $4^{\circ} \mathrm{com}$ tipos $A, B$ e $C$ (quadro 2).

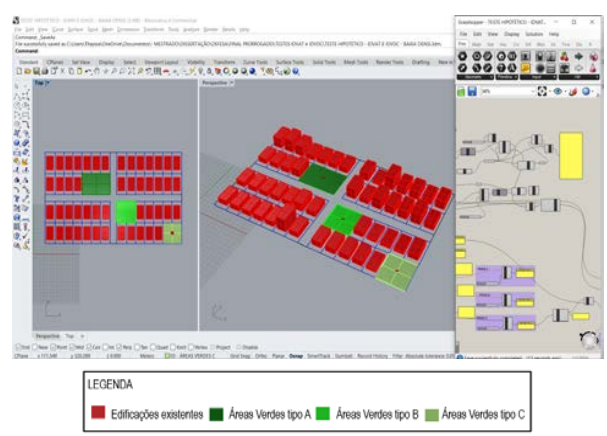

Figura 6: Modelagem Teste Hipotético 1.

\begin{tabular}{|c|c|c|c|c|c|c|}
\hline \multicolumn{7}{|c|}{ TESTE HIPOTÉTICO 1 - CENÁRIO REAL } \\
\hline \multicolumn{6}{|c|}{ INPUTS PRIMÁRIOS } & $\begin{array}{l}\text { OUTPUT/ } \\
\text { INPUT }\end{array}$ \\
\hline ÁREA & ÁREA & & & ÁREA & ÁREA & \multirow{3}{*}{$\mathrm{FSI}$} \\
\hline TOTAL DO & TOTAL & & TADA & VEGETADA & VEGETADA & \\
\hline $\begin{array}{l}\text { RECORTE } \\
\text { (em ha) }\end{array}$ & $\begin{array}{c}\text { VEGETADA } \\
\text { (em ha) }\end{array}$ & & $\begin{array}{l}\mathrm{OA} \\
\text { ha) }\end{array}$ & $\begin{array}{l}\text { TIPO B } \\
\text { (em ha) }\end{array}$ & $\begin{array}{l}\text { TIPO C } \\
\text { (em ha) }\end{array}$ & \\
\hline 2,7 & 0,30 & & & 0,08 & 0,08 & 0.70 \\
\hline \multicolumn{7}{|c|}{ OUTPUTS } \\
\hline & \multicolumn{2}{|c|}{$\begin{array}{l}\text { TESTE COM } \\
\text { TIPO A }\end{array}$} & \multicolumn{2}{|c|}{$\begin{array}{l}\text { TESTE COM } \\
\text { TIPO B }\end{array}$} & $\begin{array}{l}\text { TESTE COM } \\
\text { TIPO C }\end{array}$ & $\begin{array}{l}\text { TESTE COM } \\
\text { TIPOS A, B e C }\end{array}$ \\
\hline IDVAT & \multicolumn{2}{|c|}{0.06} & \multicolumn{2}{|c|}{0.03} & 0.01 & 0.04 \\
\hline IDVDC & \multicolumn{2}{|c|}{0.09} & & 0.04 & 0.01 & 0.05 \\
\hline
\end{tabular}

Quadro 2: Dados de saída Teste Hipotético 1.

\section{TESTE HIPOTÉTICO 2}

O segundo teste possui a mesma área total e áreas vegetadas que no teste anterior. $O$ adensamento construtivo predominante, entretanto, é de "alta densidade", definindo-se de 5 a 20 o número de pavimentos (fig. 7). Para a validação, seguiu-se a mesma lógica do teste anterior, elaborou-se 4 sub-testes, nos quais $01^{\circ}$ sub-teste considerou apenas as áreas vegetadas com predominância arbórea do tipo $A$, no $2^{\circ}$ sub-teste todas tipo $B$, no $3^{\circ}$ todas tipo $C$, no $4^{\circ} \mathrm{com}$ tipos A, B e C (quadro 3)

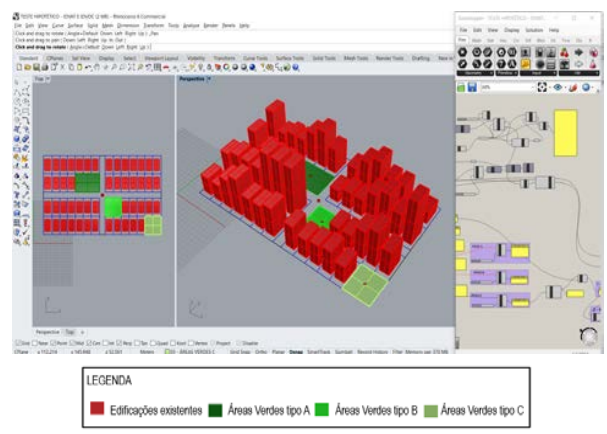

Figura 7: Modelagem Teste Hipotético 2. Fonte: Autores (2020)

\begin{tabular}{|c|c|c|c|c|c|c|}
\hline \multicolumn{7}{|c|}{ TESTE HIPOTÉTICO 2 - CENÁRIO REAL } \\
\hline \multicolumn{6}{|c|}{ INPUTS PRIMÁRIOS } & $\begin{array}{l}\text { OUTPUT/ } \\
\text { INPUT }\end{array}$ \\
\hline ÁREA & ÁREA & & & ÁREA & ÁREA & \multirow{3}{*}{$\mathrm{FSI}$} \\
\hline TOTAL DO & TOTAL & & & VEGETADA & VEGETADA & \\
\hline $\begin{array}{c}\text { RECORTE } \\
\text { (em ha) }\end{array}$ & $\begin{array}{c}\text { VEGETADA } \\
\text { (em ha) }\end{array}$ & & & $\begin{array}{l}\text { TIPO B } \\
\text { (em ha) }\end{array}$ & $\begin{array}{l}\text { TIPO C } \\
\text { (em ha) }\end{array}$ & \\
\hline 2,7 & 0,30 & & & 0,08 & 0,08 & 3.54 \\
\hline \multicolumn{7}{|c|}{ OUTPUTS } \\
\hline & \multirow{2}{*}{\multicolumn{2}{|c|}{$\begin{array}{l}\text { TESTE COM } \\
\text { TIPO A }\end{array}$}} & \multirow{2}{*}{\multicolumn{2}{|c|}{ TIPO B }} & TESTE COM & TESTE COM \\
\hline & & & & & TIPOC & TIPOSA, BeC \\
\hline IDVAT & \multicolumn{2}{|c|}{0.066} & \multicolumn{2}{|c|}{0.033} & 0.011 & 0.040 \\
\hline IDVDC & \multicolumn{2}{|c|}{0.018} & & .009 & 0.003 & 0.007 \\
\hline
\end{tabular}

Quadro 3: Dados de saída Teste Hipotético 2.

\section{TESTE HIPOTÉTICO 3}

Neste Teste 3, buscou-se elaborar um conjunto de 4 quadras, com tamanho aproximado aos testes anteriores, que se assemelham a malha urbana da cidade da cidade de Barcelona (Espanha), possuindo parte da malha urbana com alto de adensamento construtivo (fig. 8). Apresenta edificações geminadas com no máximo 10 pavimentos e miolos de quadra livres totais ou parcialmente ocupados por áreas vegetadas, sendo estes ocupando três das quatro quadras desenhadas. Da mesma forma, para 
validação, elaborou-se 4 sub-testes, nos quais o $1^{\circ}$ subteste considerou apenas as áreas vegetadas com predominância arbórea do tipo $\mathrm{A}$, no $2^{\circ}$ sub-teste todas tipo $\mathrm{B}$, no $3^{\circ}$ todas tipo $\mathrm{C}$, no $4^{\circ}$ com tipos $\mathrm{A}, \mathrm{B}$ e $\mathrm{C}$ (quadro 4 ).

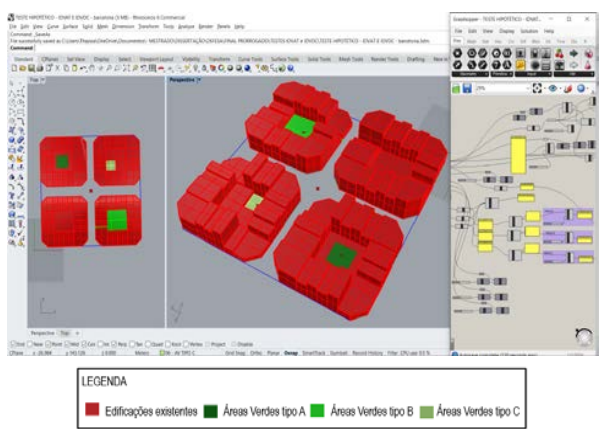

Figura 8: Modelagem Teste Hipotético 3. Fonte: Autores (2020)

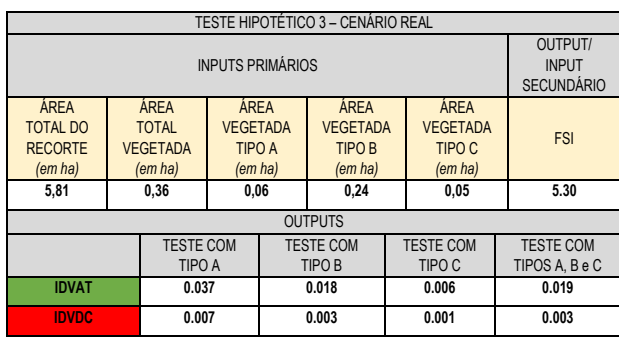

Quadro 4: Dados de saída Teste Hipotético 3. Fonte: Autores (2020)

\section{TESTE HIPOTÉTICO 4}

Na última situação hipotética, o Teste 4 detém o valor aproximado do tamanho da área total do recorte do Teste 3 , e foi esboçado semelhantemente ao padrão de adensamento construtivo das superquadras da cidade de Brasília (DF-Brasil), nas quais apresentam-se edificações soltas na escala da quadra com no máximo 6 pavimentos (fig. 9). Para validação, elaborou-se 3 sub-testes, nos quais $01^{\circ}$ sub-teste considerou apenas as áreas vegetadas com predominância arbórea do tipo $\mathrm{A}$, no $2^{\circ}$ sub-teste todas tipo $\mathrm{C}$ e no $3^{\circ}$ com tipos A e C (quadro 5).

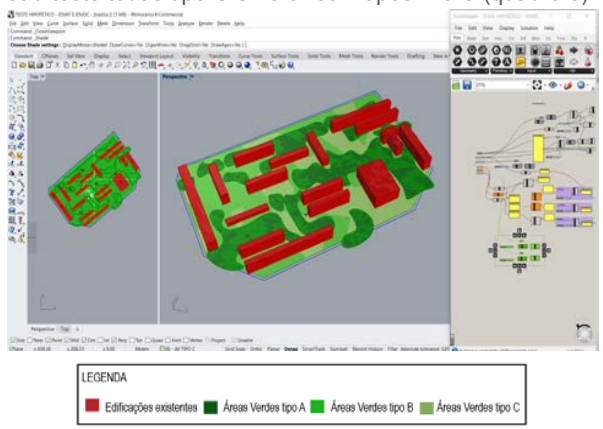

Figura 9: Modelagem Teste Hipotético 4.

${ }^{1}$ Neste momento dos Testes Hipotéticos, os indicadores estão sendo avaliados numa escala de 0 a 1.

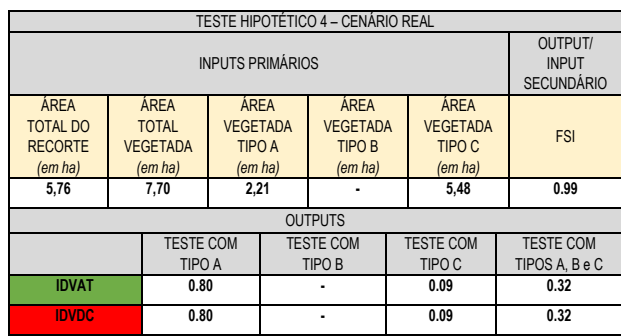

Quadro 5: Dados de saída Teste Hipotético 4.

\section{ANÁLISE DOS RESULTADOS}

Por meio da realização dos Testes Hipotéticos, assim como dos sub-testes derivados, foi possível a validação interna dos indicadores e compreensão dos valores gerados para 4 situações urbanas distintas.

Entre os Testes Hipotéticos 1 e 2, observou-se que incrementar a densidade construtiva e diminuir a densidade vegetada (do tipo A para tipo C) levou os valores dos indicadores à quase zero. Em preliminar, para se concluir algo, é preciso levar em consideração as temáticas abordadas e defendidas nesta pesquisa, na premissa de que cidades equitativamente adensadas (construção e vegetação) são mais propícias a diminuírem seu impacto aos microclimas urbanos. Por se tratar de uma validação interna, em amostragens hipotéticas, preferiu-se prosseguir sobre a possibilidade de equidade entre os dois Testes, e avaliou-se que o Teste 2 para alcançar os valores de IDVAT do Teste 1, seria preciso aumentar o tamanho das áreas destinadas ao verde de modo que estas fossem estritamente do tipo A, com maciços arbóreos desejáveis para o trabalho de mitigação do rigor climático das cidades

Os Testes Hipotéticos 2 e 3 apresentaram o maior percentual de adensamento construtivo (FSI equivalente à 3.54 e 5.3 , respectivamente), com edificações elevadas entre 5 a 20 pavimentos e recuos mínimos ou geminadas. Os Indicadores encontrados obtiveram valores baixos ${ }^{1}$, quase zero. Acredita-se que em situações de área urbana consolidada, como no caso de Barcelona (cidade na qual se inspirou o conjunto de quadras do Teste 3), o equilíbrio entre os indicadores seria talvez fosse encontrado na execução de algumas medidas legislativas como desapropriação dos miolos de quadra para incremento estritamente do verde arbóreo;

Os Testes 1 e 4 demonstram menor percentual de adensamento construtivo, com edificações elevadas até 5 pavimentos (Teste 1) e 6 pavimentos (Teste 4). O Teste 4 , tendo por base uma superquadra de Brasília, apresenta os blocos edilícios longitudinais e soltos, não no lote, mas na escala da quadra, priorizando para a presença de grupos adensados com árvores de grande porte que permeiam as edificações que contribuem para o sombreamento dos pedestres, amenização do clima e a dinâmica dos ventos pela malha urbana (ROMERO, 2011). Aplicando o IVU para essa tipologia de forma urbana e comparando com as outras amostragens testadas, observou-se o incremento dos valores dos indicadores, devido à quantidade 
adensadas de áreas vegetadas do tipo $\mathrm{A}$, sugerindo um norte para padrões equitativos entre os adensamentos construtivo e vegetado apontados pelos valores de IDVAT e IDVDC.

Os resultados foram agrupados em um gráfico de dispersão, denominado nesta pesquisa como Diagrama IVU, contendo os dois indicadores propostos. Desta forma, é possível identificar o comportamento dos valores e as correlações entre estes.

\begin{tabular}{|c|c|c|c|c|c|c|c|}
\hline \multirow{6}{*}{ 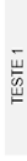 } & \multicolumn{3}{|c|}{ OUTPUTS } & \multicolumn{4}{|c|}{ OUTPUTS } \\
\hline & ÁREAS & IDVAT & IDVDC & \multirow{5}{*}{ 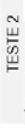 } & ÁREAS & IDVAT & IDVDC \\
\hline & TESTE TIPOA & 0,06 & 0,09 & & TESTE TIPOA & 0,066 & 0,018 \\
\hline & TESTE TIPO B & 0,03 & 0,04 & & TESTE TIPO B & 0,033 & 0,009 \\
\hline & TESTE TIPO C & 0,01 & 0,01 & & TESIE TIPO C & 0,011 & 0,003 \\
\hline & TESTE TIPO A B B e C & 0,04 & 0,05 & & TESTE TIPO A,B e C & 0,002 & 0,007 \\
\hline \multirow{6}{*}{$\stackrel{\stackrel{m}{m}}{\stackrel{m}{m}}$} & \multicolumn{3}{|c|}{ OUTPUTS } & \multicolumn{4}{|c|}{ OUTPUTS } \\
\hline & ÁREAS & IDVAT & IDVDC & \multirow{4}{*}{ 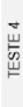 } & ÁREAS & IDVAT & IDVDC \\
\hline & TESTE TIPOA & 0,037 & 0,007 & & TESTE TIPOA & 0,8 & 0,8 \\
\hline & TESTE TIPO B & 0,018 & 0,003 & & TESTE TIPO B & - & - \\
\hline & TESTE TIPO C & 0,006 & 0,001 & & TESTE TIPO C & 0,09 & 0,09 \\
\hline & TESTE TIPO A B B C C & 0,019 & 0,003 & & TESTE TIPO $A, B$ e $C$ & 0,32 & 0,32 \\
\hline
\end{tabular}

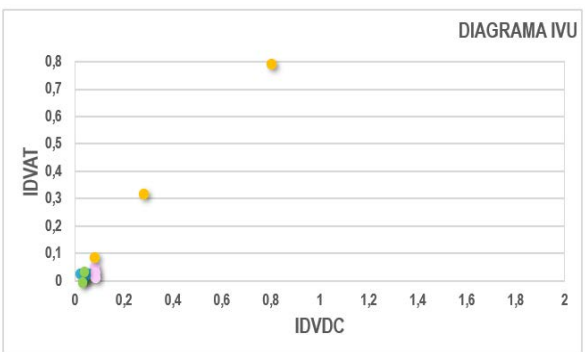

LEGENDA
Subtestes 1 Subtestes 2 Subtestes 3 Subtestes 4

Figura 10: Resultados totais dos Testes Hipotéticos e Diagrama IVU.

\section{CONCLUSÕES}

Os indícios conclusivos dos estudos de formulação, implementação e aplicação da proposta do IVU, ainda que de maneira inicial, foram obtidos de modo que se embasasse em algumas outras investigações voltadas para mensuração de parâmetros urbanísticos de densidade e vegetação urbanas (RENKE-OLIVEIRA, 1996; HARDER et al., 2005; ROSSET, 2005; BERGHAUSER-PONT \& HAUPT, 2009; CALLEJAS et. al., 2012; ARAÚJO \& FERREIRA, 2014), correlação destas mesmas variáveis (DUARTE, 2000), e de métodos de implementação computacional do espaço urbano (LIMA, 2017).

A testagem em amostragens hipotéticas, como meio de validação interna, foi, portanto, necessária para avaliação estatística dos indicadores. Assim como, devem servir de base para alguns encaminhamentos estimados, como a validação externa por meio da aplicação deste em um bairro existente, e a aferição de dados microclimáticos da região para estabelecer uma correlação entre os valores de IVU e a variação de temperatura.
O estudo de mensuração de atributos urbanos por meio da lógica algorítmica e da modelagem paramétrica mostrou sua potencialidade no que tange a possibilidade de avaliação multicenário. Neste artigo, desenhou-se os cenários reais, entretanto, é possível analisar os dados de entrada/saída em cenários tendenciais e alternativos em tempo hábil. Todavia, algumas fragilidades podem ser constatadas como o fato desta ser uma pesquisa por simulação (simulation research), ou seja, parâmetros da realidade social do recorte não são inclusos, o que poderia contribuir para qualificação dos dados.

Esta pesquisa também corrobora à esfera pedagógica no que tange ao ensino e produção acadêmica da Arquitetura e do Urbanismo. A LPV, utilizada como uma das estratégias computacionais adotadas nesta investigação, permite a implementação de estratégias de projeto generativo de modo simples para a modelagem paramétrica de situações urbanas (CELANI e VAZ, 2011) e se configura como um meio de inserção do aluno/pesquisador que não possui familiaridade com a linguagem de programação tradicional, de script.

Por fim, a metodologia proposta ainda contribui com pesquisas voltadas para a mitigação dos impactos causados pela ação antropogênica aos climas urbanos, assim como contribuir para estudos urbanos enfocados em métodos de análise que utilizam estratégias computacionais ademais das convencionais.

\section{REFERÊNCIAS}

ACIOLY, C.; DAVIDSON, F. (1998). Densidade urbana: um instrumento de planejamento e gestão urbana. Mauad Edit ed. [S.I: s.n.].

ABREU, L. V.; LABAKI, L. C. (2010). Conforto térmico propiciado por algumas espécies arbóreas: avaliação do raio de influência através de diferentes índices de conforto. Ambiente Construído, v. 10, n. 4, p. 103-117.

ARAÚJO, C. M. A.; FERREIRA, C. C. M. (2014). Áreas Verdes Públicas em Juiz de Fora (MG): Uma análise do estado da arte atual. Geo UFRJ, n. 1415-7543, 2014.

BARBIRATO, G. M.; BARBOSA, R. V. R.; TORRES, S. C. (2012). Articulação entre clima urbano e planejamento das cidades: velho consenso, contínuo desafio. In: $8^{\circ}$ Congreso Internacional Ciudad y Territorio Virtual, Río de Janeiro, 10, 11 y 12 Octubre. Centre de Política de Sòl i Valoracions.

BEIRÃO, J. (2012). CltyMaker: designing grammars for urban design. TU Delft.

CALLEJAS, I., CAMPOS, A., Durante, L. C., \& NOGUEIRA, M. (2012). Índices arbóreos e suas relações com o microclima urbano. Encontro Nacional de Tecnologia do Ambiente Construído (ENTAC), Juiz de Fora (MG), 14.

CELANI, G. (2011). Algorithmic Sustainable Design: Uma visão crítica do algoritmo generativo. Arquitextos. 
Disponível

em:

https://www.vitruvius.com.br/revistas/read/resenhasonline/ $10.116 / 3995$

CELANI, G.; VAZ, C. (2012). CAD scripting and visual programming languages for implementing computational design concepts: A comparison from a pedagogical point of view. International Journal of Architectural Computing, v. 10, n. 1, p. 121-137.

FERREIRA, C. de C. M. (2014). Modelo para análise das variáveis de cobertura da terra e a identificação de microclimas, em centros urbanos. Revista Brasileira de Climatologia, v. 14, n. 1.

FERREIRA, C. C. M.; MONTEIRO, A.; PAULA, I. F. M. (2019). Áreas verdes e desigualdades sociais em um município de médio porte no Brasil. Caderno de Geografia, v. 29, n.56.

GOMES, P. S.; LAMBERTS, R. (2009). O estudo do clima urbano e legislação urbanística: considerações a partir do caso Montes Claros-MG. Ambiente Construído, v. 9, n. 1, p. 73-91.

HENKE-OLIVEIRA, C. (1996). Planejamento ambiental na Cidade de São Carlos (SP) com ênfase nas áreas públicas e áreas verdes: diagnósticos e propostas. $196 \mathrm{f}$. Dissertação de Mestrado. Universidade Federal de São Carlos (SP).

BERGHAUSER-PONT, M.; HAUPT, P. (2009) Space, Density and Urban Form. Doctoral thesis. Delft University of Technology.

DUARTE, D. H. S. (2000). Padrões de ocupação do solo e microclimas urbanos na região de clima tropical continental. Tese de Doutorado. Universidade de São Paulo. 2000.

DUARTE, D. H. S.; SERRA, G. G. (2003). Padrões de ocupação do solo e microclimas urbanos na região de clima tropical continental brasileira: correlações e proposta de um indicador. Ambiente construído, v. 3, n. 2.
Intergovernmental Panel on Climate Change (IPCC). (2016). Climate Change and Land. International Panel of Climate Changes.

LABAKI, L. C.; SANTOS, R. F. S.; BUENOBARTHOLOMEI, C. L.; ABREU, L. V. (2011). Vegetação e conforto térmico em espaços urbanos abertos. Fórum Patrimônio, v. 4, n. Mudanças climáticas e o impacto das cidades.

LIMA, F. T. A. (2017). Métricas Urbanas: Sistema (para)métrico para análise e otimização de configurações urbanas de acordo com métricas de avaliação de desempenho. Doutorado em Urbanismo, Universidade Federal do Rio de Janeiro.

LOMBARDO, M. A. Ilha de calor nas metrópoles: o exemplo de São Paulo. Editora Hucitec com apoio de Lalekla SA Comércio e Indústria, 1985.

NEVES, T. B. S. (2020). Indicadores para o Verde Urbano (IVU): Proposta de uma implementação computacional. Dissertação de Mestrado em Arquitetura e Urbanismo, Universidade Federal da Paraíba.

NUCCI, J. C. (2001). Qualidade Ambiental E Adensamento Urbano. [S.I: s.n.], 2001.

ROSSET, F. (2005). Procedimentos metodológicos para estimativa do Índice de Áreas Verdes Públicas. Estudo de caso: Erechim (RS) . Dissertação de Mestrado em Ecologia e Recursos Naturais, Universidade Federal de São Carlos.

SILVA, B. A.; XAVIER, T. C.; SILVA, F. T.; ALVAREZ, C. E. (2015). O impacto da distribuição de vegetação no microclima de ambientes urbanos. Proceedings of EURO ELECS 2015. Guimarães, Portugal.

SPANGENBERG, J. (2009). Retro-innovating Nature in Megacities: São Paulo/Brazil-a case-Study. Tese de Doutorado em Engenharia, Universidade Bauhaus de Weimar. 